\title{
Enhancement of Second Harmonic Signal in Nanofabricated Cones
}

\author{
F. Dutto, ${ }^{\dagger}$ M. Heiss, ${ }^{\dagger}$ A. Lovera, ${ }^{\S}$ O. López-Sánchez, ${ }^{\|}$A. Fontcuberta i Morral, ${ }^{\ddagger}$ and A. Radenovic ${ }^{*}, \dagger$ \\ ${ }^{\dagger}$ Laboratory of Nanoscale Biology, Institute of Bioengineering, ${ }^{\ddagger}$ Laboratory of Semiconductor Materials, Institute of Materials , \\ ${ }^{\S}$ Laboratory of Nanophotonics and Metrology, Institute of Micro Engineering, and "Laboratory of Nanoscale Electronics and \\ Structures, Institute of Electrical Engineering, School of Engineering, EPFL, 1015 Lausanne, Switzerland
}

\section{Supporting Information}

ABSTRACT: Geometrical effects in optical nanostructures on nanoscale can lead to interesting phenomena such as inhibition of spontaneous emission, ${ }^{1,2}$ high-reflecting omnidirectional mirrors, structures that exhibit low-loss-waveguiding, ${ }^{3}$ and light confinement. ${ }^{4,5}$ Here, we demonstrate a similar concept of exploiting the geometrical effects on nanoscale through precisely fabricating lithium niobate $\left(\mathrm{LiNbO}_{3}\right)$ nanocones arrays devices. We show a strong second harmonic generation (SHG) enhancement, shape and arrangement dependent, up to 4 times bigger than the bulk one. These devices allow below diffraction limited observation, being perfect platforms for
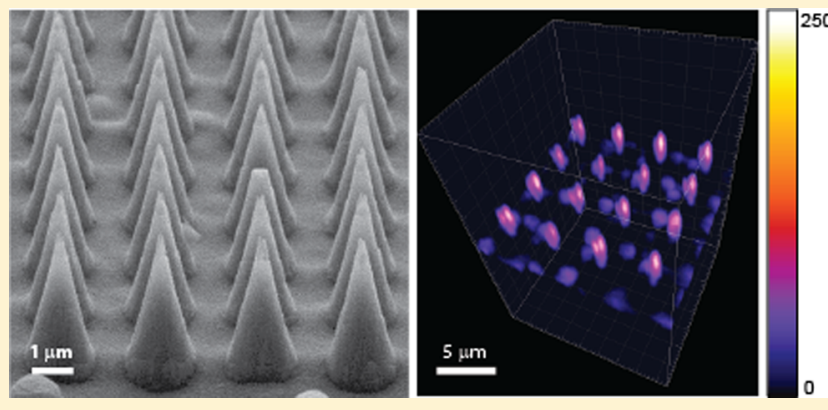
single molecule fluorescence microscopy ${ }^{6}$ or single cell endoscopy. ${ }^{7}$ Nanocones create a confined illumination volume, devoid from blinking and bleaching, which can excite molecules in nanocones proximity. Illumination volume can be increased by combining the SH enhancement effect with plasmon resonances, excited thanks to a gold plasmonic shell deposited around the nanostructures. This results in a local further enhancement of the SH signal up to 20 times. The global SH enhancement can be rationally designed and tuned through the means of simulations.

KEYWORDS: Second harmonic generation, lithium niobate, nanocones, enhancement, plasmons, single molecule detection

$\mathrm{N}$ owadays, frequency conversion is a common tool used to create visible coherent light for the frequencies where no alternative laser sources are available. At the heart of this phenomenon are typically nonlinear birefringent crystals, which respond to the interaction with sufficiently high light intensities with nonlinear optical processes. Efficient second order nonlinear optical interactions, ${ }^{8}$ such as second harmonic generation (SHG), sum frequency generation, and difference frequency generation, occur only in noncentrosymmetric crystals, which are crystals that do not display inversion symmetry in their unit cell. Over the years, it has been realized that for the highest efficiency of the SHG process, employed crystals with dimensions much larger than the impinging laser wavelength have to be phased matched. On the other hand, in nanoscale materials, which has one of the structure dimensions much smaller than the coherent length, the phase matching condition can usually be ignored. ${ }^{9}$ Additionally, thanks to their limited size, light-emitting nanoscale probes have been proposed as the ideal platform for single molecule fluorescence imaging ${ }^{6}$ and single molecule endoscopy. ${ }^{7}$ SHG nanostructures perfectly match the requirements for these applications, having already been used as local excitation sources. ${ }^{10,11}$ They were also used as probes for the cell and in vivo imaging ensuring relatively low intensity illumination of cells that avoids cell damage and being free from photobleaching, photoblinking, and dye saturation effects. ${ }^{12,13}$ Today, a reliable fabrication method allowing for parallel and high signal-to-noise ratio measurement is still missing.
In principle and, as we are going to demonstrate, biocompatible $\mathrm{LiNbO}_{3}$ structures, as ultrasharp $\mathrm{LiNbO}_{3}$ microtips arrays reported by Mailis et al., ${ }^{14}$ constitute the perfect system for these applications. Our nanocones arrays are almost 1 order of magnitude smaller and denser than Mailis's structures, allowing for higher parallelization.

Although in noncentrosymmetric materials SHG is quite intense, it is well-known that nonlinear optical effects are relatively weak processes. There is a constant search for ways of improving the efficiency of the $\mathrm{SH}$ process, and in the last few decades plasmons (resonating at the $\mathrm{SH}$ frequency) were widely exploited to further increase the photon-photon interactions that governed the $\mathrm{SH}^{15-17}$ Two types of plasmons can be excited in thin metal surfaces or in metal nanoparticles: in the first case, surface electromagnetic waves propagating at the metal-dielectric interface are called surface plasmon polaritons (SPPs), whereas in the second case, the localized surface plasmons (LSPs) sustained by nanoparticles (thus size and shape dependent) are excited by the resonant wavelength. Here, we report the $\mathrm{SH}$ emission of arrayed $\mathrm{LiNbO}_{3}$ nanocones and we discuss the effect of a uniform thin gold ( $\mathrm{Au}$ ) layer, 30 $\mathrm{nm}$ thick, covering the structures. (Evaporation thickness is nominally $30 \mathrm{~nm}$ on the substrate but it can be as low as $6.5 \mathrm{~nm}$ on the nanocones walls.) The thickness of the layer was chosen

Received: September 2, 2013

Revised: November 15, 2013

Published: November 21, 2013 

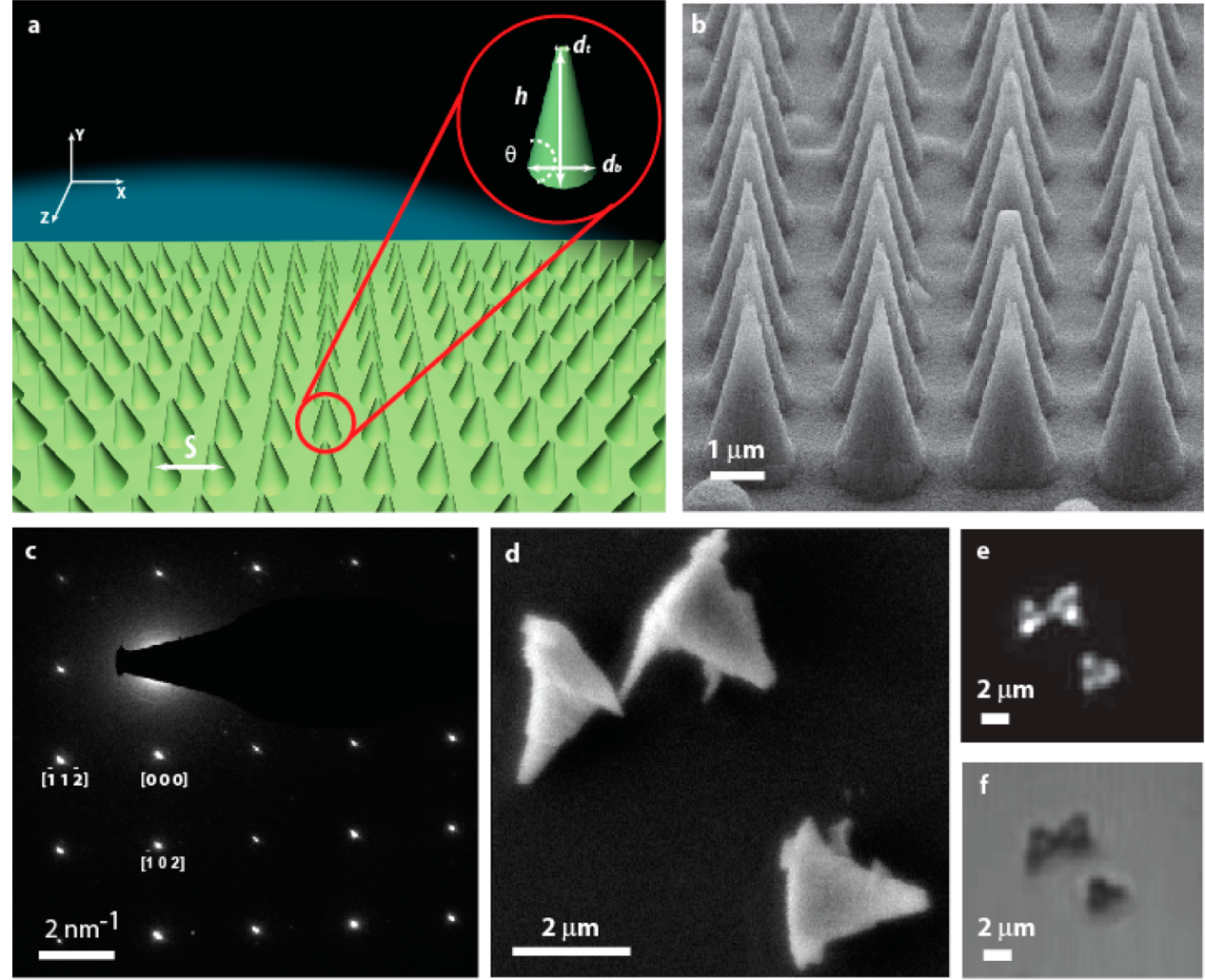

Figure 1. (a) Schematic representation of the $\mathrm{LiNbO}_{3}$ nanocones on a Y-cut substrate. E-beam lithography allows control over the pitch $S$ between the nanocones and the top nanocone diameter $d_{\mathrm{v}}$, while the angle $\theta$ and the height of the nanocones $h$ are predetermined by the etching properties of $\mathrm{LiNbO}_{3}$. (b) Tilted SEM image $\left(60^{\circ}\right)$ of lithographically fabricated $\mathrm{LiNbO}_{3}$ arrayed nanocones. (c) $\mathrm{LiNbO}_{3}$ nanocone TEM selected area electron diffraction pattern. Indexing has been performed by using the JEMS software. ${ }^{41}$ (d) SEM image of extracted $\mathrm{LiNbO}_{3}$ nanocones. (e) Two photon confocal image (excitation power set at $3 \mathrm{~mW}$ ) of $\mathrm{LiNbO}_{3}$ nanocones shown in panel d. (f) Bright-field image of the extracted $\mathrm{LiNbO}_{3}$ nanocones shown in panel $\mathrm{d}$.

to coat the structures with the thinnest possible layer on the nanocones walls that would still support plasmon resonances $^{16,17}$ but that it is still thin enough on the top of the nanocones to minimize the absorption of the exciting beam. Traditionally, in SHG applications lithium niobate $\left(\mathrm{LiNbO}_{3}\right)$ and potassium dihydrogen phosphate (KDP) were the most commonly used nonlinear birefringent crystals, but strong SHG signals have been reported in many other types of single inorganic nanostructured materials including $\mathrm{GaN}^{18}{ }^{18} \mathrm{GaAs},{ }^{19}$ $\mathrm{ZnO}{ }^{20}$ and $\mathrm{X}-\mathrm{NbO}_{3}{ }^{10,11,21-23}$ nanowires, $\mathrm{BaTiO}_{3}$ nanocryst$\mathrm{als}^{12,13,24}$ and InAs nanomembranes. ${ }^{25}$ So far, many reported nanomaterials that show high SHG are mostly chemically synthesized $^{10-13,18,26}$ leading to samples with low uniformity in terms of dimensions, shapes, structures, and properties with difficulty to be patterned in the desired arrangement. Here, we report how top-down approach can be used to fabricate welldefined nanostructures starting from $\mathrm{LiNbO}_{3}$ wafers.

$\mathrm{LiNbO}_{3}$ has been one of the most widely used and versatile nonlinear optical materials due to several attractive properties, for example, high nonlinear coefficients $\left(d_{33}=25.2 \mathrm{pm} / \mathrm{V}\right.$ at $\lambda=$ $1064 \mathrm{~nm}^{27}$ ), wide transparency range, availability of substrates of large size, and high homogeneity. $\mathrm{LiNbO}_{3}$ lithographically fabricated structures of fine features are rarely reported ${ }^{28-32}$ because of the relatively difficult etching of this brittle and inert material. In this paper, we present steps that allow the fabrication of arrays of nanocones with moderate aspect ratios and tip diameters. Nanolithography tools, such as e-beam lithography, allowed the creation of arrays of nanocones with various pitch $S$ and top nanocone diameter width $d_{\mathrm{v}}$, whereas the etching process and shadow mask thickness predetermined the nanocones height $h$ and the angle of nanocones walls $\theta^{31,32}$ (see schematic in Figure 1a). Details on the fabrication process are reported in Supporting Information. We used scanning electron microscopy (SEM) imaging to inspect fabricated nanostructures. As shown in Figure $1 b$, we structured the samples in nanocones arrays of similar dimensions with a welldefined geometrical arrangement. For further SEM and transmission electron microscopy (TEM) sample inspections, $\mathrm{LiNbO}_{3}$ nanocones can be shaved off from the chip by gently scraping the surface with a clean microtome blade (Ultratrim, Diatome), then dispersed in deionized water, and deposited on an imaging sample or grid. TEM imaging was performed to examine the status of the crystal after fabrication process and in particular that it has not been altered. Figure 1c shows a diffraction pattern taken by TEM in which the presence of clear diffraction spots indicates that the sample kept its original crystallinity. By measuring the distances between the spots of the diffraction pattern, any stress or defect created into the crystal can be detected as a mismatch in the measured spots positions with respect to the theoretical pattern. The perfect match between the measurement and theory eliminates the possibility of any crystal modification due to the fabrication steps.

Subsequently, samples were coated with $30 \mathrm{~nm}$ thick gold layer in order to examine the influence of this thin film on the SHG emission. Coating has been performed with a thermal 
evaporation system (Leybold Optics LAB 600H) that creates a very uniform $30 \mathrm{~nm}$ layer on flat surfaces, while a thinner layer, ranging from 6.5 to $10.5 \mathrm{~nm}$ (depending on the samples geometries), on the nanocones side walls.

Once the conservation of the crystalline structure is confirmed, excitation and emission of $\mathrm{SH}$ signals from the nonlinear $\mathrm{LiNbO}_{3}$ nanostructures have been tested. We used two-photon confocal microscope with femtosecond laser pulsed excitation, centered at $\lambda=854 \mathrm{~nm}$ for SHG measurement. The photons collection window has been centered at $\lambda=427 \pm 10$ $\mathrm{nm}$ in order to collect only photons originated from SHG process. We employed a $63 \times$ water immersion objective with a numerical aperture (NA) of 1.2. Figure 1d shows a SEM picture of three extracted nanocones then observed with the twophoton confocal microscope (see Figure 1e,f). The whole imaging method is detailed in Supporting Information. Cones removed from the substrate present local enhancements in the $\mathrm{SH}$ signal (see Figure 1e) along their axis due to Fabry-Pérot cavity modes supported by the nanostructures. SHG intensity $I_{2 \omega}$ stored inside the cavity, for different cavity squared cross section dimension $d$, can be easily related to the fundamental wavelength intensity $I_{\omega}$ using the simple expression $I_{2 \omega} \alpha I_{\omega}^{2}$. The fundamental wavelength intensity is obtained by using the following formula ${ }^{33}$

$$
I_{\omega}(d)=I_{0} \frac{1}{1+F \sin ^{2}\left[\frac{\phi(d)}{2}\right]}
$$

where $F=4 R /\left(1-R^{2}\right)^{2}$ is the finesse, $R$ is the reflectivity and has been determined through the use of Fresnel equations, $\phi(d)=2 \pi \Delta s / \lambda$ is the phase difference, $\Delta s=2 n_{2} d \cos \beta$ is the path difference, $n_{2}$ is the $\mathrm{LiNbO}_{3}$ refractive index, and $\beta$ is the angle of refraction. Maxima in the signal are obtained when the phase difference is an integer multiple of $2 \pi$ thus for $d=m \lambda$ / $\left(2 n_{2} \cos \beta\right)$ with $m=1,2,3, \ldots$ Because $\mathrm{LiNbO}_{3}$ is a negative uniaxial birefringent material, it supports two different refractive indexes for different crystal orientation such as ordinary $\left(n_{\mathrm{o}}=\right.$ 2.49 at $854 \mathrm{~nm})$ and extraordinary $\left(n_{\mathrm{e}}=2.17\right.$ at $\left.854 \mathrm{~nm}\right)$ refractive indexes. Birefringence needs to be taken into account in this calculation thus both ordinary and extraordinary refractive indexes of $\mathrm{LiNbO}_{3}$ are considered, leading to a definition of a range of cross section dimensions supporting the cavity modes. Resonances are expected at multiple of the cross section dimensions $d \sim m \cdot[205-214] \mathrm{nm}$ leading to broad and unresolvable peaks because of our diffraction limited optics. Moreover, low nanocones facets reflectivity $R \sim[11-16] \%$ results in moderate intensity peaks. However, in Figure 1e two intensity peaks in the SHG signal are observed at the two extremities of the cones probably originate from the additional ray reflections formed at the top and bottom surfaces facets leading to more intense modes.

The strong enhancement effect presented above is also observed in arrayed nanocones. In contrast to the extracted nanocones, in every arrayed nanocone, fabricated on the same substrate, crystal orientation is identical and allows for systematic investigation of the influence of different sample geometries and different nanocones arrangements on the $\mathrm{SH}$ emission. Since the $\mathrm{SH}$ signal is orientation dependent, it results in a different behavior for different sample cuts and different beam polarization orientations. Knowing that the highest $\mathrm{LiNbO}_{3}$ SHG coefficient is along $Z$-axis $\left(d_{33}=17 \mathrm{pm} /\right.$ $\left.\mathrm{V}^{34}\right)$, the highest emission is detected whenever the laser polarization is parallel to it. The polarization in the two-photon microscope, usually set to horizontal $\left(0^{\circ}\right.$, p-polarized light $)$, can only be rotated along the horizontal plane, thus the alignment condition is fulfilled only in $\mathrm{X}$ and $\mathrm{Y}$ samples cuts. Polarization dependence of $\mathrm{Y}$ cut sample SH signal is reported in Figure 2a
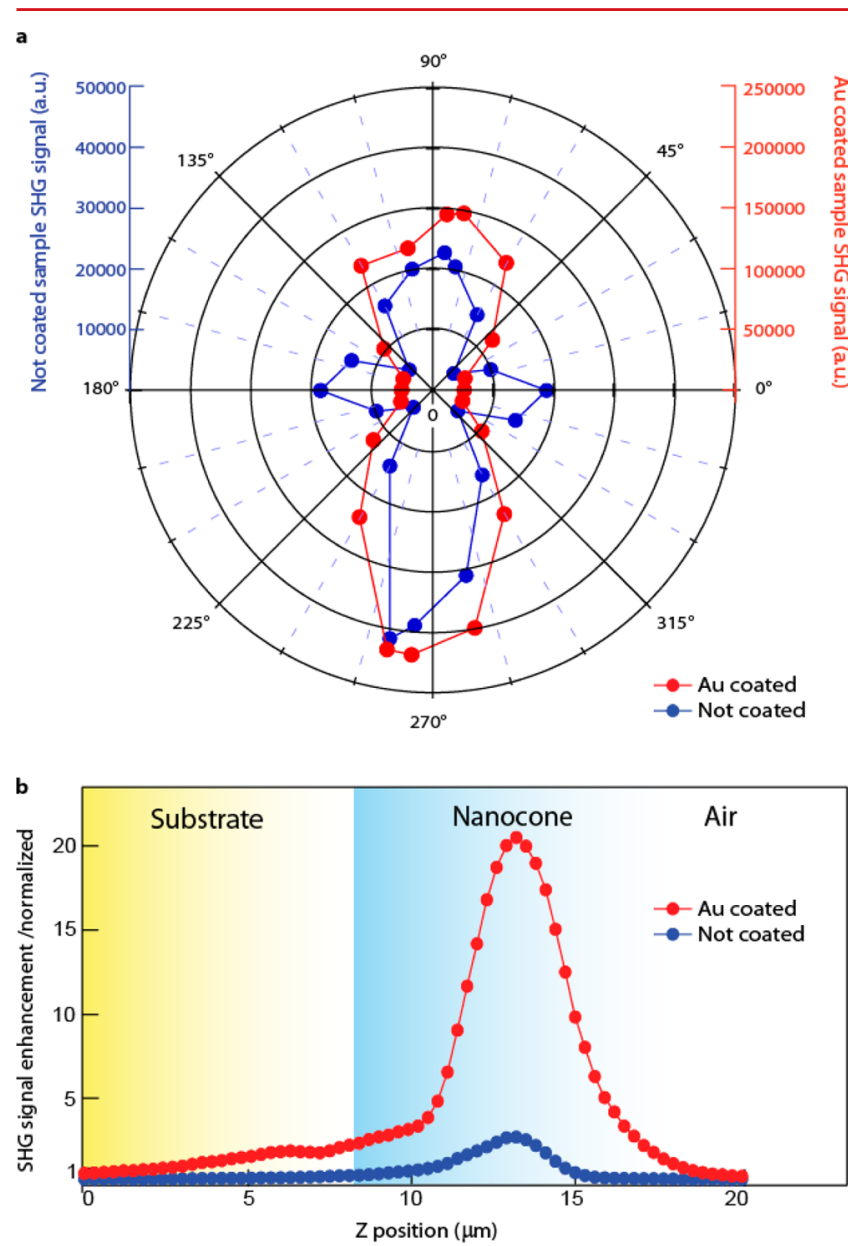

Figure 2. (a) $\mathrm{SH}$ polar graphs versus input polarization of bare $\mathrm{LiNbO}_{3}$ nanocones and gold coated ones. Reported intensities are expressed in arbitrary units. (b) Z-profiles of SH signals from bare $\mathrm{LiNbO}_{3}$ nanocones and gold-coated ones. Profiles have been normalized in respect to the $\mathrm{LiNbO}_{3}$ substrate (not structured) value. Nanocone position can be recognized by the presence of the peaks in the Z-profiles.

(blue trace) showing a four lobes behavior typical of $\mathrm{LiNbO}_{3}$ with the highest response for polarization at $270^{\circ}$. Prior to all measurements, we performed a quick polarization scan to find the optimal polarization angle at which the highest SHG response is detected. This angle may vary from sample to sample due to limited precision of placing the sample under the microscope.

The maximum SH signal values, obtained from different fabricated samples scanned in three dimensions, are reported in Table S1 of Supporting Information. We measured a SH enhancement up to 4 times the bulk value in the cones. Enhancement on restricted regions of the samples makes room for applications requiring a confined volume illumination. Moreover one should consider that $\mathrm{LiNbO}_{3}$ presents a low cytotoxicity thus a good biocompatibility ${ }^{35}$ and that nanocone arrays can support cell cultures (data not shown here). As suggested by Xie et al. ${ }^{6}$ for silicon dioxide nanopillars, our nanocones are an ideal platform for single molecule 

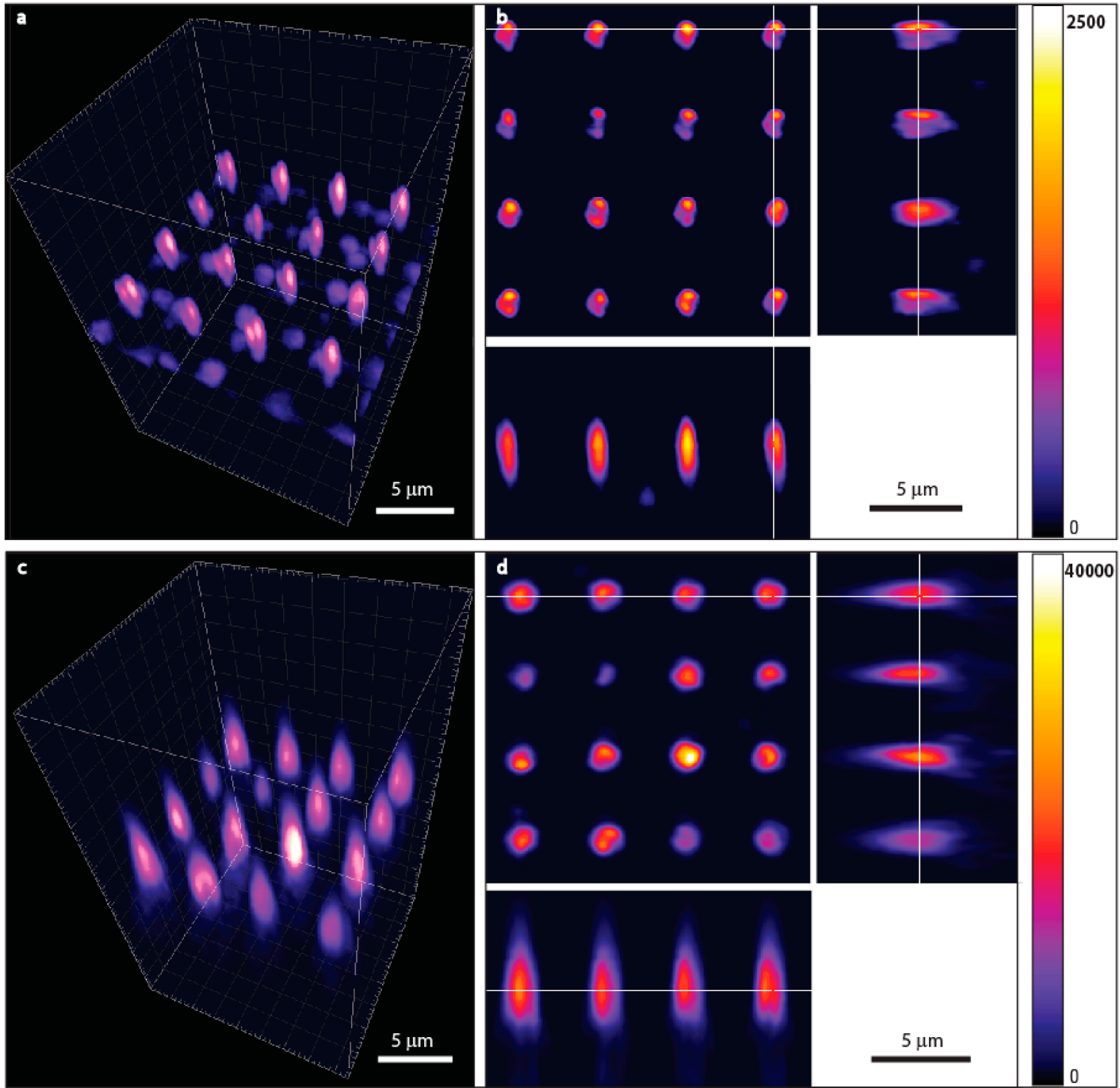

Figure 3. (a) A $3 \mathrm{D}$ image of $\mathrm{SH}$ signal generated by a $\mathrm{Y}$-cut $\mathrm{LiNbO}_{3}$ sample. (b) A $2 \mathrm{D}$ sections of $\mathrm{SH}$ signal generated by the sample shown in panel a. The scale is adapted to show nanocones SHG enhancement respect to the substrate emission set to zero. (c) A 3D image of SH signal generated by the sample shown in panel a coated with $30 \mathrm{~nm}$ of gold. (d) A 2D sections of SH signal generated by the sample shown in panel c. The scale is adapted to show Au-coated nanocones SHG enhancement respect to the substrate emission not covered by gold. Note that the saturation of the SH signal has been avoided by increasing drastically the maximum intensity of the scale bar in respect to the one in panels a and $b$.

fluorescence imaging. The high fabrication precision and control and the array arrangement improve the technique allowing parallelized measurements and a deep confined volume excitation.

Fabricated samples with and without gold coating have been measured in three dimensions with the two-photon confocal microscope. Samples have been sandwiched between a glass slide and a glass coverslip. The point spread function (PSF) of the two-photon excitation laser-scanning microscope is wide and affects the resolution along the three axes. In our system, the lateral resolution is approximately $300 \mathrm{~nm}$ whereas the axial one is approximately $1200 \mathrm{~nm}$. Figure $2 \mathrm{~b}$ shows the typical normalized SHG enhancement Z-profiles of a Y-cut $\mathrm{LiNbO}_{3}$ sample coated and not coated with gold. A clear peak is present in both traces in conjunction with the nanocone position showing a strong enhancement of the $\mathrm{SH}$ signal emitted by the nanocones respect to the substrate one. By nanostructuring a $\mathrm{LiNbO}_{3}$ substrate, one creates a big enhancement of $\mathrm{SH}$ signal ranging from 150 up to $400 \%$. An even larger enhancement is observed in coated structures in which the SHG is drastically increased by coupling the cavities to plasmonic effects. By adding the Au layer, plasmons are excited and the signal is in fact further increased from 8 to more than 20 times. Not normalized $3 \mathrm{D}$ profiles of the same samples are shown in Figure 3. In all panels of Figure 3, the substrate SHG contribution has been set at 0 (black in the picture) and the bright spots are the $\mathrm{SH}$ emission coming from nanocones regions. To show the enhancement effect in both configurations, the maximum intensity has been set at 2500 for the uncoated (bare) sample (Figure 3a,b) and at 40000 (Figure $3 \mathrm{c}, \mathrm{d})$ for the gold-coated structure. Localization of the nanocones is possible from the $\mathrm{SH}$ signal image. The spectra of structured and unstructured samples in both bare and coated configurations, as reported in Supporting Information Figure S4, confirm that the observed enhancement is an effect of the plasmons resonating at the $\mathrm{SH}$ frequency. Gaussian fits of spectra from bare $\mathrm{LiNbO}_{3}$ substrate, and bare and coated nanocone show a peak centered at the $\mathrm{SH}(428 \mathrm{~nm})$ 
wavelength, while the unstructured sample coated with gold is not showing any peak.

Meanwhile, plasmonic contribution is mainly influenced by gold surface, quality, uniformity, and roughness, which displays a large variability in the emitted signal in the presence of surface defects. One should also consider that, under a certain power density, the very thin gold coating can be melted by the strongly focused laser beam. For all samples, melting was clearly visible on structures illuminated with more than $10 \mathrm{MW} / \mathrm{cm}^{2}$, thus the employed laser power density was always kept below this limit. However, melting can be induced by local ununiformity of the gold layer, thus this effect can also arise at lower laser power densities $\left(5 \mathrm{MW} / \mathrm{cm}^{2}\right)$. Another effect of the coating is the change in the SHG polarization dependence as it is shown in Figure $2 \mathrm{a}$ (red trace). The gold coating acts as a polarizer for the SHG signal, cutting one polarization component and transmitting the other. This silencing effect of SHG, which depends on the impinging polarization direction, has been previously observed in simpler structures such as coupled antenna; ${ }^{36}$ the SHG in the gap of a coupled antenna was shown to be silenced for longitudinal excitation ( $p$ polarized light) and not for transversal excitation (s-polarized light). Here we report the same effect. The transmitted polarization component corresponds to the best polarization orientation of the bare sample.

Now we change gears to understand the enhanced SHG. Sample geometry strongly affects the enhancement of both bare and coated samples. However arrangement influence is harder to be detected because of the quite narrow beam waist (around $500 \mathrm{~nm}$ ) of our multiphoton confocal microscope not allowing the simultaneous excitation of many cones per scanned point. Only partial excitation of neighboring cones is possible at certain positions, showing a higher $\mathrm{SH}$ signal for smaller pitch (data are reported in Table S1 of Supporting Information). On a large scale, this effect could be demonstrated with an epiillumination system. We thus address theoretically the influence of nanocones arrangement on the $\mathrm{SH}$ signal emission by simulating the $\mathrm{LiNbO}_{3}$ samples excited by a plane wave. Light matter interaction in standing nanocones is a complex phenomenon with a strong dependence on the nanocone dimensions, shape, and absorption coefficient of the raw material. ${ }^{37-39}$ We have performed 3D finite difference time domain simulations with $\mathrm{MEEP}^{40}$ in order to obtain the electric field strength inside the nanocone arrays. For the simulation, we consider an array unit cell with periodic boundary conditions in $x$ and $y$ that is illuminated by a plane wave from the top. For simplicity, the crystal orientation of $\mathrm{LiNbO}_{3}$ substrate is not taken into account in simulations. In Figure 4, we report modeling results, which show the typical spatial distribution of the square of the electric field modulus in a cross section of a cone. One should know that in a nonplanar nanoscale cone array the light polarization inside the cones does not directly relate to the polarization of the far-field laser beam but also strongly depends on the cone geometry and the coupling of a plane wave to the cones. The cone shape geometry and arrangement result in a significant electric field enhancement in each cone at very localized positions up to a factor of about 1300 for given geometries (see Table S1 of Supporting Information). Overall, the average enhancement from the whole volume is more than 6 times larger than the bulk $\mathrm{SH}$ signal. Given the fact that light extraction is improved in the cone geometry, this enhancement constitutes very good

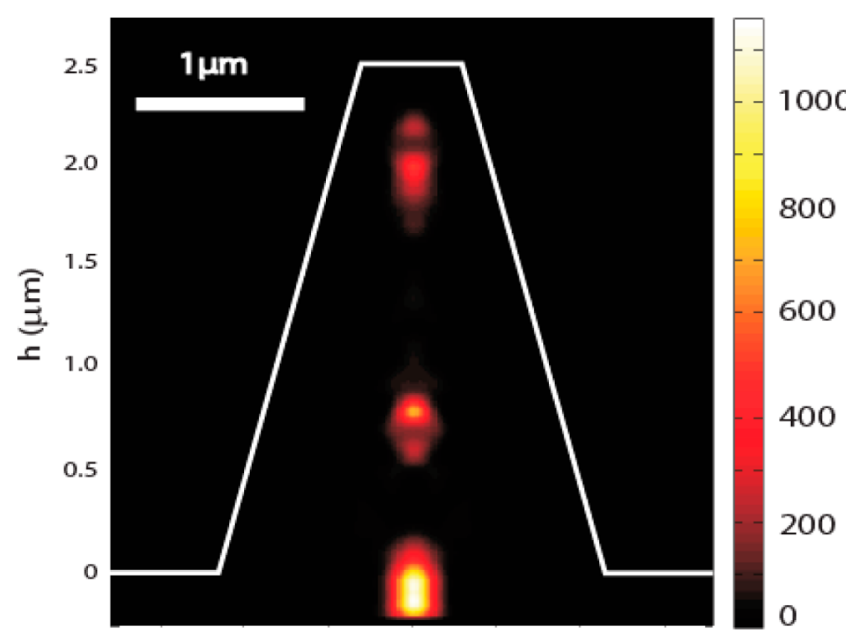

Figure 4. Simulated SHG intensity cross section of a Y-cut $\mathrm{LiNbO}_{3}$ nanocone in its arrayed form. Local enhancement peaks are visible along the cone axis.

news for the use of cones as localized submicrometer SHG sources.

Even if the ideal geometry for SHG enhancing would be the nanowire one (data not shown here), nanowires are not producible with the fabrication technique presented here because of technological limitations related to the fixed etching angle that do not allow the fabrication of structures with vertical walls. As a consequence, the spacing between fabricated nanocones cannot be smaller than a certain value and it has to obey to the following equation: $S>\left(h / \mathrm{e} \theta+d_{\mathrm{e}}\right)$. Only samples that could be realized experimentally are taken into account in simulations. Table S1 in Supporting Information reports the structural parameters of some samples that have been realized and their respective simulated average and local $\mathrm{SH}$ enhancements. Also theoretically, we observed that there is an increase in $\mathrm{SH}$ efficiency due to the nanocones arrangement that is inversely proportional to the pitch; $\mathrm{SH}$ signal is increased by almost two times in the fabricated sample with the smallest spacing with respect to the one with the biggest one. The obtained simulations results are very promising and make room for improvement. Further modeling would be very useful to find the best structure dimensions and arrangement that maximize the $\mathrm{SH}$ enhancement contributions coming from nanocones dimensions and arrangement and plasmons effects. Moreover, improvement in the fabrication processes can also lead to less rough surfaces and smaller structures thus, possibly, further increase the enhancement.

Conclusion. In summary, we reported several interesting points. We have shown a top-down technique to fabricate $\mathrm{LiNbO}_{3}$ nanocones with constant walls incline and varying spacing. Structuring the substrate creates nanocones FabryPérot cavities that result in a strong SHG enhancement in both extracted and arrayed nanocones. An enhancement of the $\mathrm{SH}$ nanocones signal with respect to the bulk one up to 4 times is reached with certain nanocones geometries and arrangements. Furthermore, SH signal can be drastically increased by coating the sample with a thin gold layer hence by exciting plasmon resonances. A total $\mathrm{SH}$ enhancement up to 20 times is obtained.

Finally, we have performed simulations on arrayed nanocones illuminated from the top by a plane wave showing that the shape and the arrangement of nanocones are influencing 
the SH emission. Our simulations will open the way for the optimal design of future nanophotonic devices. SH enhancement effects that have been demonstrated in this work can be further maximized by refining some fabrication steps such as electroplating and etching and by additionally studying the best possible configuration to have the highest contribution from all reported effects. To conclude we presented a very versatile nanophotonic device that allows local multifocal excitation in the visible range and a wide number of possible applications. The good fabrication control, the strong emitted signal, and the big excitation volume makes it a good platform for fluorescence imaging or as portable multifocal microscope in endoscopy applications.

\section{ASSOCIATED CONTENT}

\section{S Supporting Information}

Fabrication details, confocal characterization, polarization and optical spectrum measurements, Supplementary Figures 1-4, and Supplementary Table 1. This material is available free of charge via the Internet at http://pubs.acs.org.

\section{AUTHOR INFORMATION}

\section{Corresponding Author}

*E-mail: aleksandra.radenovic@epfl.ch.

\section{Author Contributions}

A.R., A.F.iM., F.D., and M.H. designed the study. F.D. fabricated the samples, performed SHG measurements, and analyzed the data presented in the paper and Supporting Information. F.D. and A.R prepared the manuscript. M.H. performed the simulations presented in the paper and prepared the relative section of the manuscript together with A.F.iM. A.L. helped F.D. with the data analysis and with the understanding of the whole phenomena. O.L.S. performed with F.D. spectra measurements not presented in the paper. All authors read and commented on the manuscript.

\section{Notes}

The authors declare no competing financial interest.

\section{ACKNOWLEDGMENTS}

This work was supported by Swiss National Science Foundation (FNS) with Grants 200021-125319, NCCRQSIT, and Synergia CRSII3_132396/1 and by ERC with Grant UpCon. We thank Camille Raillon for her help in the fabrication process, Willyan Hasenkamp for the design of the electroplating setup and access to it, Gwenn Ulliac from FEMTO-ST for having performed the dry etch step, the Centre Interdisciplinaire de Microscopie Electronique (CIME) at EPFL for access to the electron microscopes and Bioimaging and Optics Platform (PTBIOP), and in particular Thierry Laroche for access to confocal microscope and continuous support. Many thanks to Romain Guiet and Olivier Burri for their help in image processing and the helpful discussions. Thanks as well to Jérémy Butet and Krishnan Thyagarajan for their interest in the project and their help. We finally thank Esther Alarcon-Llado for her help with the optical system for spectral analysis, and Sylvie Roke, Carlos Macias-Romero, and Rüdiger Scheu for having tested one of our samples with their optical setup.

\section{REFERENCES}

(1) Bulgarini, G. Spontaneous emission control of single quantum dots in bottom-up nanowire waveguides. Appl. Phys. Lett. 2012, 100, 121106.

(2) Leistikow, M. D. Inhibited Spontaneous Emission of Quantum Dots Observed in a 3D Photonic Band Gap. Phys. Rev. Lett. 2011, 107, 193903 .

(3) Ghoshal, S. K.; Sahar, M. R.; Rohani, M. S.; Sharma, S. In Nanophotonics for 21st Century; Padmanabhan, P., Ed.; InTech: New York, 2011.

(4) Chia Wei, H.; et al. Observation of trapped light within the radiation continuum. Nature 2013, 499, 188-191.

(5) Krogstrup, P.; et al. Single-nanowire solar cells beyond the Shockley-Queisser limit. Nat. Photonics 2013, 7, 306-310.

(6) Xie, C.; Hanson, L.; Cui, Y.; Cui, B. X. Vertical nanopillars for highly localized fluorescence imaging. Proc. Natl. Acad. Sci. U.S.A. 2011, 108, 3894-3899.

(7) Yang, P. D.; Yan, R. X.; Fardy, M. Semiconductor Nanowire: What's Next? Nano Lett. 2010, 10, 1529-1536.

(8) Franken, P. A.; Weinreich, G.; Peters, C. W.; Hill, A. E. Generation of Optical Harmonics. Phys. Rev. Lett. 1961, 7, 118.

(9) Wiederrecht, G. Handbook of nanoscale optics and electronics; Elsevier: New York, 2009.

(10) Nakayama, Y.; et al. Tunable nanowire nonlinear optical probe. Nature 2007, 447, 1098-U1098.

(11) Dutto, F.; Raillon, C.; Schenk, K.; Radenovic, A. Nonlinear Optical Response in Single Alkaline Niobate Nanowires. Nano Lett. 2011, 11, 2517-2521.

(12) Hsieh, C. L.; Grange, R.; Pu, Y.; Psaltis, D. Bioconjugation of barium titanate nanocrystals with immunoglobulin $\mathrm{G}$ antibody for second harmonic radiation imaging probes. Biomaterials 2010, 31, 2272-2277.

(13) Pantazis, P.; Maloney, J.; Wu, D.; Fraser, S. E. Second harmonic generating (SHG) nanoprobes for in vivo imaging. Proc. Natl. Acad. Sci. U.S.A. 2010, 107, 14535-14540.

(14) Mailis, S. et al. In Lasers and Electro-Optics, (CLEO), Conference on Lasers and Electro-Optics, Baltimore, Maryland, May 22, 2005; pp $232-234$.

(15) Agarwal, G. S.; Jha, S. S. Theory of 2nd Harmonic-Generation at a Metal-Surface with Surface-Plasmon Excitation. Solid State Commun. 1982, 41, 499-501.

(16) Kauranen, M.; Zayats, A. V. Nonlinear plasmonics. Nat. Photonics 2012, 6, 737-748.

(17) Pu, Y.; Grange, R; Hsieh, C. L.; Psaltis, D. Nonlinear Optical Properties of Core-Shell Nanocavities for Enhanced Second-Harmonic Generation. Phys. Rev. Lett. 2010, 104, 207402.

(18) Long, J. P.; Simpkins, B. S.; Rowenhorst, D. J.; Pehrsson, P. E. Far-field imaging of optical second-harmonic generation in single GaN nanowires. Nano Lett. 2007, 7, 831-836.

(19) Grange, R; et al. Far-Field Imaging for Direct Visualization of Light Interferences in GaAs Nanowires. Nano Lett. 2012, 12, 54125417.

(20) Johnson, J. C.; et al. Near-field imaging of nonlinear optical mixing in single zinc oxide nanowires. Nano Lett. 2002, 2, 279-283.

(21) Dutto, F.; Radenovic, A. Alkaline niobate nanowires as optomechanical probes. Proc. SPIE 2012, 8458, 84581X.

(22) Sergeyev, A.; et al. Second-harmonic generation in lithium niobate nanowires for local fluorescence excitation. Opt Express 2013, 21, 19012-19021.

(23) Grange, R.; Dutto, F.; Radenovic, A. In Nanowires Implementations and Applications; Hashim, A., Ed.; Intech: New York, 2011, Chapter 23.

(24) Kim, E.; et al. Second-Harmonic Generation of Single $\mathrm{BaTiO}_{3}$ Nanoparticles down to $22 \mathrm{~nm}$ Diameter. ACS Nano 2013, 7 (6), 5343-5349.

(25) Pecora, E. F.; et al. Enhanced second harmonic generation from InAs nano-wing structures on silicon. Nanoscale 2013, 5, 1016310170 . 
(26) Bonacina, L.; et al. Polar Fe(IO3)(3) nanocrystals as local probes for nonlinear microscopy. Appl. Phys. B: Lasers Opt. 2007, 87, 399-403.

(27) Shoji, I.; Kondo, T.; Kitamoto, A.; Shirane, M.; Ito, R. Absolute scale of second-order nonlinear-optical coefficients. J. Opt. Soc. Am. B 1997, 14, 2268-2294.

(28) Solntsev, A. S. et al. Cascaded third harmonic generation in lithium niobate nanowaveguides. Appl. Phys. Lett. 98 (2011).

(29) Hartung, H.; et al. Ultra thin high index contrast photonic crystal slabs in lithium niobate. Opt. Mater. 2010, 33, 19-21.

(30) Benchabane, S.; Robert, L.; Rauch, J. Y.; Khelif, A.; Laude, V. Highly selective electroplated nickel mask for lithium niobate dry etching. J. Appl. Phys. 2009, 105 (9), 094109-094109-6.

(31) Queste, S. et al. Deep reactive ion etching of quartz, lithium niobate and lead titanate. JNTE Proceedings; Toulouse: France, 2008.

(32) Queste, S.; Ulliac, G.; Jeannot, J.-C.; Khan Malek, C. In Proceedings of the 4th international conference on multi-material micro manufacture, Conference Multi-Material Micro Manufacture, Cardiff, U.K., Sep 9-11, 2008; pp 9-11.

(33) Demtröder, W. Laser spectroscopy, 4th ed.; Springer: New York, 2008.

(34) Arizmendi, L. Photonic applications of lithium niobate crystals. Phys. Status Solidi A 2004, 201, 253-283.

(35) Staedler, D.; et al. Harmonic Nanocrystals for Biolabeling: A Survey of Optical Properties and Biocompatibility. ACS Nano 2012, 6, 2542-2549.

(36) Berthelot, J.; et al. Silencing and enhancement of secondharmonic generation in optical gap antennas. Opt. Express 2012, 20, 10498-10508.

(37) Tsakalakos, L. Strong broadband optical absorption in silicon nanowire films. J. Nanophotonics 2007, 1, 013552 .

(38) Zhu, J.; et al. Optical Absorption Enhancement in Amorphous Silicon Nanowire and Nanocone Arrays. Nano Lett 2009, 9, 279-282.

(39) Mariani, G.; et al. Patterned Radial GaAs Nanopillar Solar Cells. Nano Lett 2011, 11, 2490-2494.

(40) Oskooi, A. F.; et al. MEEP: A flexible free-software package for electromagnetic simulations by the FDTD method. Comput. Phys. Commun. 2010, 181, 687-702.

(41) Stadelmann, P. A. JEMS http://cimewww.epfl.ch/people/ stadelmann/jemsWebSite/jems.html (accessed Sep 21, 2004). 